Kong. Res. J. 3(1) : 73-75, 2016 ISSN 2349-2694

Kongunadu Arts and Science College, Coimbatore.

\title{
GREEN ROUTE SYNTHESIZED SILVER NANOPARTICLES: AS POTENTIAL ANTIBACTERIAL MATERIAL
}

\author{
Rajmohan, $\mathrm{D}^{\mathbf{1}^{*} \text {,, R. Ranjithkumar }{ }^{2}, \text { K. Logankumar }{ }^{1}, \text { P. Sagadevan }}{ }^{3}$, B. Chandrashekar ${ }^{4}$ and R. Yamuna ${ }^{1}$ \\ ${ }^{1}$ Department of Zoology, Kongunadu Arts and Science College, Coimbatore. \\ ${ }^{2}$ Department of Biotechnology, Dr.N.G.P. Arts and Science College, Coimbatore. \\ 3PG \& Research Department of Biotechnology, Kongunadu Arts and Science College, Coimbatore. \\ ${ }^{4}$ Department of Physics, Nanotechnology Research Lab, Kongunadu Arts and Science College, Coimbatore. \\ *E. mail: rajmohandevadass@gmail.com
}

\begin{abstract}
The green-synthesized method is rapid, superficial, toxic free, suitable, less time consuming, environmental safe and can be applied in a variety of applications in medicinal field. In the present study demonstrated antibacterial action of Vinca rosea Leaf extract medicated Silver Nanoparticles (VrL-AgNPs) tested against both Gram positive and Gram negative pathogens such as Staphylococcus aureus, Escherchia coli and Pseudomonas aeruginosa. The obtained results indicate the VrL-AgNPs achieved maximum zone of inhibition against test pathogens P. aeruginosa and significant action against E.coli and S. aureus. These green routes synthesized silver nanoparticles using biological sources like plant and plant extract which makes them a potent source of antibacterial agent.
\end{abstract}

Keywords: AgNPs, VrL, Antibacterial, ZOI, pathogenic.

\section{INTRODUCTION}

Nanotechnology is fast growing by producing nanoproducts and nanoparticles that can have novel and size-associated physico-chemical properties differing considerably from larger matter. The new properties of nanoparticles have been subjugated in a broad range of potential applications in cosmetics, renewable energies material, medicine, biomedical devices and environmental remediation (Tran et al., 2013). Metals like gold, silver, platinum and zinc have been used for the biosynthesis of nanoparticles having greater potentials application in field of nanotechnology. Among the all metal nanoparticles, the silver nanoparticles (nanosilver or AgNPs) have increasing attention due to their unique chemical, physical and biological properties. Silver nanoparticles are very important and most widely used nanoparticles with potential applications in biomedical nanotechnology (Arunachalam et al., 2013).

The emerging infectious diseases and the development of drug resistance in the pathogenic bacteria and fungi at an alarming rate is a matter of serious concern. Despite the increased knowledge of microbial pathogenesis and application of modern therapeutics, the morbidity and mortality associated with the microbial infections still remains high (Kolar et al., 2001). Therefore, there is a pressing demand to discover novel strategies and identify new antimicrobial agents from natural and inorganic substances to develop the next generation of drugs or agents to control microbial infections. Prior to the extensive use of chemotherapeutics in modern health care system, inorganic antimicrobials such as silver and copper were used since ancient times to treat microbial infections (Moghimi, 2005). In the recent times, the advances in the field of nanosciences and nanotechnology has brought to fore the nanosized inorganic and organic particles which are finding increasing applications as amendments in industrial, medicine and therapeutics, synthetic textiles and food packaging products (Gajjar et al., 2009; Silvestre et al., 2011). This is present study deals with antibacterial properties Vinca rosea Leaf (VrL) extract medicated Silver Nanoparticles (VrL-AgNPs) using Vinca rosea plant extract tested against both Gram positive and Gram negative pathogens.

\section{MATERIALS AND METHODS}

\subsection{Green Synthesis VrL-AgNP}

Our earlier study reported that the simple and environmental free green route was used to synthesis AgNPs from silver nitrate using aqueous extract of Vinca rosea (VrL) leaf. VrL extract mediated synthesized AgNPs was characterized using by UV visible spectroscopy (UV-Vis), X-ray Diffraction Spectrum (XRD), Scanning Electron 
Microscopy (SEM) and Energy Dispersive X-ray Analysis (EDX) and obtained VrL-AgNPs showed spherical in shape with average particles size round around 30- $70 \mathrm{~nm}$ (Rajmohan et al., 2015). These green synthesized VrL-AgNPs powder sample was tested for antibacterial activity against human pathogens.

\subsection{Microorganisms used}

Human pathogenic bacteria culture such as Staphylococcus aureus (S. aureus) Escherchia coli (E. coli) and Pseudomonas aeruginosa (P. aeruginosa) were obtained from Kovai Medical Center Research and Hospital (KMCH) Coimbatore, Tamilnadu, India.

\subsection{Antibacterial Activity of VrL-AgNPs}

Green route synthesized VrL-AgNPs were tested for antibacterial activity by standard disc diffusion method against three different human pathogenic bacteria. In brief, pure culture of bacteria strain on Mueller Hinton Agar (MHA), the bacterial test organisms were grown in Nutrient Broth (NB) at $37^{\circ} \mathrm{C}$ for 24 hours. Followed by this step, about 100 $\mu \mathrm{l}$ of aliquot of each strain $\left(1 \mathrm{X} 10^{5} \mathrm{cfu} / \mathrm{mL}\right)$ was spread uniformly onto the individual plates using sterile cotton swabs and allowed to dry for 15-30 minutes. On other hand, VrL-AgNPs stock prepare at concentration of $0.5 \mathrm{mg} / \mathrm{mL}$ followed this, sterile filter paper discs around $5 \mathrm{~mm}$ in diameter were loaded with $100 \mu \mathrm{l}$ of VrL-AgNPs (Fig.1) and reference drug were placed on the each cultured plate and incubated at optimum temperature $\left(37^{\circ} \mathrm{C}\right)$ for 24 hours to 48 hours and the diameters of the inhibition zones were measured in millimeters.

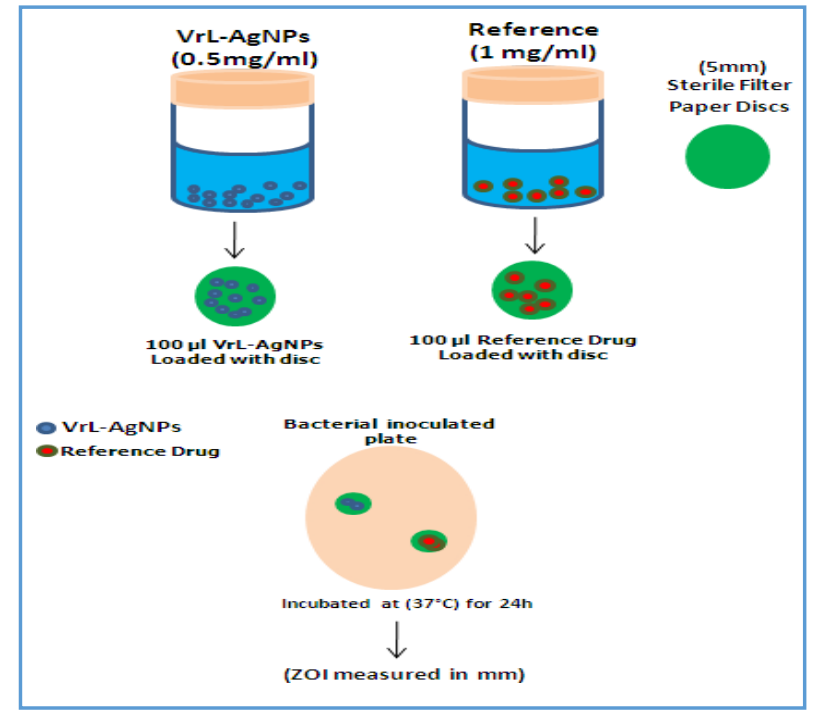

Fig. 1. Schematic diagram of preparation of VrLAgNPs

\section{RESULTS AND DISCUSSION}

\subsection{Antibacterial Activity}

Drug resistant pathogens are more pathogenic microorganisms with high mortality rate than that of wild strain. To overcome microbial drug resistant, researcher are looking forward for the development of alternative, low cost and environmental free novel drugs. Nanobiotechnology is filed of biology with nanotechnology expected to open some new aspects to fight and prevent diseases using atomic scale tailoring of materials (Afreen et al., 2011). Antibacterial activity of silver is wellknown for many years (Kamyar et al., 2012). The inhibitory action of silver compounds and silver ions had been historically recognized and applied as a useful therapeutic agent for preventing wound infections (Gordon et al., 2010). In this study, antibacterial activity of green route synthesized VrLAgNPs was evaluated by using standard Zone of Inhibition (ZOI) microbiology assay. Significant antibacterial properties against various pathogens was investigated and compared with control, the diameter of inhibition zones increased for the test pathogen (Fig. 2).

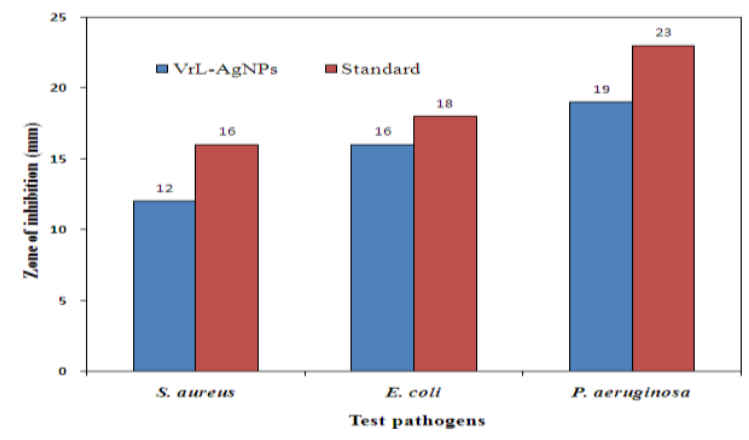

Fig. 2. Antibacterial activity of VrL-AgNPs against different human pathogens

VrL-AgNPs shown maximum ZOI was found to be $19 \mathrm{~mm}$ for $P$. aeruginosa, whereas, the other two bacteria strains of $a$. aureus and E. coli showed ZOI of 12 and $16 \mathrm{~mm}$. The standard antibiotic streptomycin obtained 16, 18 and $23 \mathrm{~mm}$ against $S$. aureus, E. coli and P. aeruginosa respectively (Fig. 3). Ranjithkumar et al. (2013) study reported that the nanocomposite (BN-AgNPs) showed excellent antibacterial activity against both gram negative $E$. coil and Gram-positive $S$. aureus. In this present study indicated the good antibacterial activity of plant mediated VrL-AgNPs showed inhibition zone against all the studied bacteria and achieved maximum activity against $P$. aeruginosa $(19 \mathrm{~mm})$. 


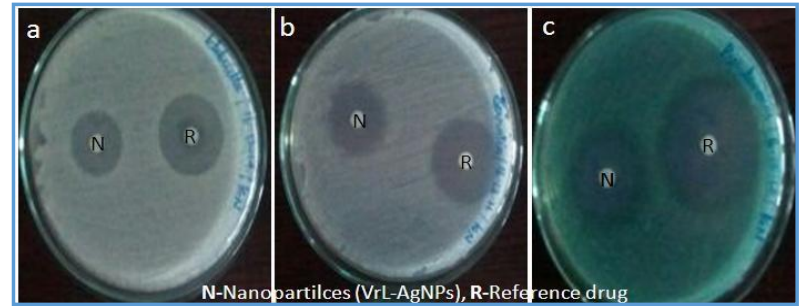

Fig. 3. Antibacterial activity of VrL-AgNPs.

a) S. aureus, b) E. coil and c) P. aeruginosa

Many researchers demonstrated the green route synthesis of silver nanoparticles by using bacteria, actinomycetes, fungi and plants. However, the plant materials (leaf, stem, seeds, fruit, roots and peels) have been successfully used for silver nanoparticles production because to their potential medicinal property, huge availability, faster rate of synthesis and toxicity free (Ranjithkumar et al., 2013; Shanmugavadivu et al., 2014). The inhibitory mechanisms of silver on bacterial cells are related to the interaction of AgNPs with thiol groups present in respiratory enzymes in bacteria. Whereas, Nano size crystalline AgNPs shows the most effective inhibitory properties with a rapid inhibition rate against human pathogenic bacteria (Wright et al., 1998). In the present study results indicated that the plant extract mediated VrL-AgNPs have potential antibacterial action against test human pathogens.

\section{CONCLUSION}

The antibacterial activities of green route synthesized silver nanoparticles were evaluated against human pathogenic bacteria such as S.aureus, E.coli and P.aeruginosa. The VrL-AgNPs showed excellent antibacterial action against P.aeruginosa and moderated action against other test human pathogens. Present study results demonstrated that biosynthesized silver nanoparticle, as a kind of antibacterial material, has a great promise for application in a wide range of biomedical applications.

\section{REFERENCES}

Afreen, V. Rathod and E. Ranganath, (2011). Synthesis of monodispersed silver nanoparticles by Rhizopus stolonifer and its antibacterial activity against MDR strains of Pseudomonas aeruginosa from burnt patients. Int. J. Environ. Sci. 1:1583-1592.

Arunachalam, K.D., S. K. Annamalai, A.M. Arunachalam, K. Subashini and S. Kennedy, (2013). Green synthesis of crystalline silver nanoparticles using Indigofera aspalathoides - medicinal plant extract for wound healing applications. Asian J. Chem. 25:311-314

Gajjar, P., B. Pettee, D.W. Britt, W. Huang, W.P. Johnson and J. Anderson, (2009). Antimicrobial activities of commercial nanoparticles against an environmental soil microbe, Pseudomonas putida KT2440. J. of Biol. Eng. 3:9-22.

Gordon, O., T. Vig Slenters, P.S. Brunetto, A.E. Villaruz and D.E. Sturdevant, (2010) Silver coordination polymers for prevention of implant infection: thiol interaction, impact on respiratory chain enzymes, and hydroxyl radical induction. Antimicrob. Agents Chemother. 54:4208-4218.

Kamyar Shameli, Mansor Bin Ahmad, Seyed Davoud Jazayeri , Parvaneh Shabanzadeh , Parvanh Sangpour, Hossein Jahangirian and Yadollah Gharayebi, (2012). Investigation of antibacterial properties silver nanoparticles prepared via green method. Chem. Cent. J. 6(73):1-10.

Kolar, M., K. Urbanek and T. Latal, (2001). Antibiotic selective pressure and development of bacterial resistance. Int. J. Antimicrob. Ag. 17:357-363.

Moghimi, S.M., (2005).Nanomedicine: prospective diagnostic and therapeutic potential. Asia Pacific Biotech. News. 9:1072-1077.

Rajmohan, D., D. Saranya, K. Logankumar, R. Ranjithkumar and B. Chandrashekar, (2015). Biomimetic Synthesis and Characterization of Silver Nanoparticles (AgNPs) Using Vinca Rosea Aqueous Extract, Kong. Res. J. 2(2): 1-5.

Ranjithkumar Rajamani, Selvam Kuppusamy and M. Shanmugavadivu, (2013). Green Synthesis of Silver Nanoparticles Using Areca Nut Extract for Enhanced Antibacterial Activity. J. Green Sci. Technol. 1, 102-106.

Ranjithkumar Rajamani, Selvam Kuppusamy, and M. Shanmugavadivu (2013). Antibacterial Textile Finishing via Green Synthesized Silver Nanoparticles, J. Green Sci. Technol. 1:111-113.

Shanmugavadivu, M., Selvam Kuppusamy and R. Ranjithkumar, (2014). Synthesis of Pomegranate Peel Extract Mediated Silver Nanoparticles and its Antibacterial Activity, Am. J. Adv. Drug Del. 2(2), 174-182.

Silvestre, C., D. Duraccio and C. Sossio, (2011). Food packaging based on polymer nanomaterials, Prog. Polymer Sci. 36:1766-1782.

Tran, Q.H., V.Q. Nguyen and A.T. Le, (2013). Silver nanoparticles: synthesis, properties, toxicology, applications and perspectives. Adv. Nat. Sci.: Nanosci. Nanotechnol. 4:1-20.

Wright, J.B., K. Lam and R.E. Burrell, (1998). Wound management in an era of increasing bacterial antibiotic resistance: a role for topical silver treatment. Am. J. Infect. Control 26:572-577. 